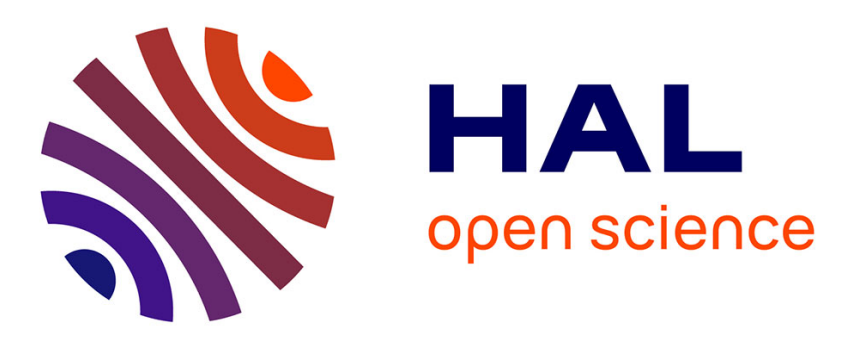

\title{
USER CONTEXT MODELS : A FRAMEWORK TO EASE SOFTWARE FORMAL VERIFICATIONS
}

\author{
Amine Raji, Philippe Dhaussy
}

\section{To cite this version:}

Amine Raji, Philippe Dhaussy. USER CONTEXT MODELS : A FRAMEWORK TO EASE SOFTWARE FORMAL VERIFICATIONS. 12th International Conference on Enterprise Information Systems, Jun 2010, Funchal, Madeira, Portugal. pp.1. hal-00471931

\section{HAL Id: hal-00471931 https://hal.science/hal-00471931}

Submitted on 9 Apr 2010

HAL is a multi-disciplinary open access archive for the deposit and dissemination of scientific research documents, whether they are published or not. The documents may come from teaching and research institutions in France or abroad, or from public or private research centers.
L'archive ouverte pluridisciplinaire HAL, est destinée au dépôt et à la diffusion de documents scientifiques de niveau recherche, publiés ou non, émanant des établissements d'enseignement et de recherche français ou étrangers, des laboratoires publics ou privés. 


\title{
USER CONTEXT MODELS : A FRAMEWORK TO EASE SOFTWARE FORMAL VERIFICATIONS
}

\author{
Amine Raji, Phillipe Dhaussy \\ LISyC-ENSIETA, 2 rue François Verny 29806, Brest Cedex 9, France \\ Université Européenne de Bretagne, Brest, France \\ amine.raji@ensieta.fr, dhaussy@ensieta.fr
}

Keywords: Formal verifications, Context Description Language, model transformation, User Context Models, Property specification patterns.

\begin{abstract}
Several works emphasize the difficulties of software verification applied to embedded systems. In past years, formal verification techniques and tools were widely developed and used by the research community. However, the use of formal verification at industrial scale remains difficult, expensive and requires lot of time. This is due to the size and the complexity of manipulated models, but also, to the important gap between requirement models manipulated by different stackholders and formal models required by existing verification tools. In this paper, we fill this gap by providing the UCM framework to automatically generate formal models used by formal verification tools. At this stage of our work, we generate behavior models of environment actors interacting with the system directly from an extended form of use cases. These behavioral models can be composed directly with the system automata to be verified using existing model checking tools.
\end{abstract}

\section{INTRODUCTION}

Verification of software systems is an important task that aims to check whether design meets intended requirements. Formal methods have demonstrated their potential in this area especially through the so called model checking. However, the application of such techniques in industrial practices is still limited w.r.t the growing need of quality and reliability of developed software.

In this paper we present our approach to overcome this shortcoming by presenting a model based approach to bridge the gap between high level requirement models and models required by existing formal verification tools. The proposed approach aims to smooth the way properties and contexts ${ }^{1}$ are derived from requirement documents and specifications.

We previously presented CDL (Context description language)(Dhaussy et al., 2008) to fill

\footnotetext{
${ }^{1}$ by contexts we refer to interactions that happen between the system under study and its environment
}

the gap between user models ${ }^{2}$ and formal models required to perform formal verifications. CDL is presented in the form of UML like graphical diagrams (subset of activity and sequence diagrams) to capture environment interaction. Additionally, a textual syntax is prosed to formalize properties to be check using property description patterns (Dwyer et al., 1999). CDL was evaluated through several aeronautic and military industrial case studies (Dhaussy et al., 2009). Conclusions of this evaluation is that CDL considerably helps practitioners to formally verify whether designed models meet intended requirements.

However, CDL is a low level language that requires design details since early development phases to produce precise enough specifications for formal verifications. We are currently working on an intermediate concept called UCM (User Context Models). UCM is thought to be user oriented and aims to utilize models constructed during development phases to automatically generate

\footnotetext{
${ }^{2}$ models manipulated during development phases of the development process
} 
formal models (i.e CDL models). The idea is to encourage users to put more details and avoid ambiguities during their modeling activities to automatically derive formal specifications.

This paper is organized as follow: Section 2 presents a background on CDL models and context aware verification techniques. Section 3 details the UCM framework and argues how they can be useful to fill the gap between requirement specification and formal verification activities. Section 4 presents related works and Section 5 discusses future work and draws some conclusions.

\section{BACKGROUND}

In software verification, model checking is increasingly used to verify that a formally specified model satisfy some desired property (Lamsweerde, 2009). In the most frequent form of model checking, the inputs to the checker are a formal state machine model and a desired property formalized in temporal logic. Applying this technique in an industrial context suffer from the combinatorial explosion induced by the internal complexity of the software to be verified.

One way to circumvent this problem consists of specifying/restricting the context in which the system will be used. This context corresponds to well-defined operational phases, such as, for example, initialization, reconfiguration, degraded mode, etc. The system is then tightly synchronized with its environment so that properties can be checked in specific contexts which limits the size of the generated state space.

In this context, CDL aims to ease the construction of context models and to specify properties. CDL describes a system environment using activity and sequence diagrams, together with a textual syntax. Activity and sequence diagrams are used to describe contexts, and the textual syntax to describe properties to be checked. The properties are specified using property description patterns and attached to specific elements in the activity diagram describing the context.

CDL have demonstrated its usefulness in porting formal verifications into industrial practices through several industrial projects (Dhaussy et al., 2009). However, CDL models construction remains a manual process requiring time and efforts to understand the system specifications in order to produce precise contexts' interactions.

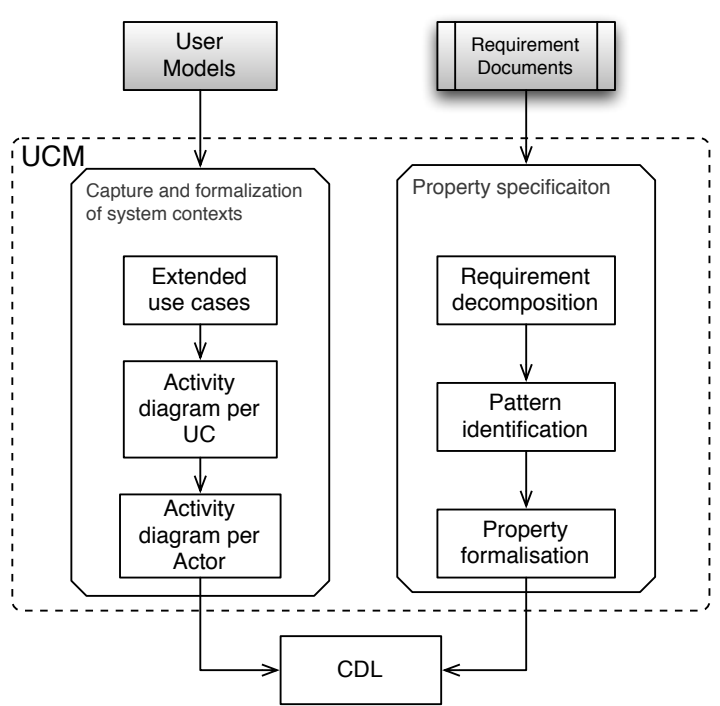

Figure 1: UCM content overview

\section{USER CONTEXT MODELS}

The main idea is to encourage engineers to put enough details in their daily constructed models. This encouragement is made through the UCM framework that presents guidelines for constructing contexts and specifying properties as well as algorithms to automate formal models generations from these models. Figure 1 shows an overview of UCM content. The left side of figure 1 presents different steps leading to the generation of formal models describing the behavior of different actors of the environment, i.e. context. The right side shows the property specification activities that aims to formalize properties to be checked.

We have defined and formalized the generation of behavioral models of the context using a model based approach (the left side of figure 1). We use an extended form of use cases to capture system requirements as well as possible exceptions and corresponding handlers if any. The idea behind this step is to gather all useful information about the system behavior and its interactions with environment actors. Constructed use cases are then used as input in our model transformation to generate formal models directly processable by a model checker.

Extended use cases are similar to traditional use cases except that they capture system requirements as well as possible exceptions and corresponding handlers if any (Mustafiz et al., 2009).In fact, many exceptional situations might appear 
during the execution of an application. The difficulties arising during verification process are usually related to the missing of relevant information about system behavior, especially when an exception endangers the normal execution of a use case. To encounter this problem, we propose that engineers (whom designed the system in the first place) specify their systems using extended use cases to foresee these exceptional situations and document how the system should deal with them. This approach leads engineers to systematically investigate all possible exceptions arising in the environment that the system may be exposed to.

We have proposed a metamodel of the extended use cases (figure 2) and an algorithm to derive the behavior of different actors involved in the use case.

In figure 2, classes with a white background are imported from the UML metamodel, and should be related to the identical ones presented in (OMG, 2007). The classes with the filled background was introduced to deal with exceptions and handlers in traditional use cases. The important point in the proposed metamodel is that main scenario steps have to be described in a structured natural language to be transformed into actions in the generated activity diagram. Used structured language is RDL (Requirement Description Language) introduced in (Nebut et al., 2003).

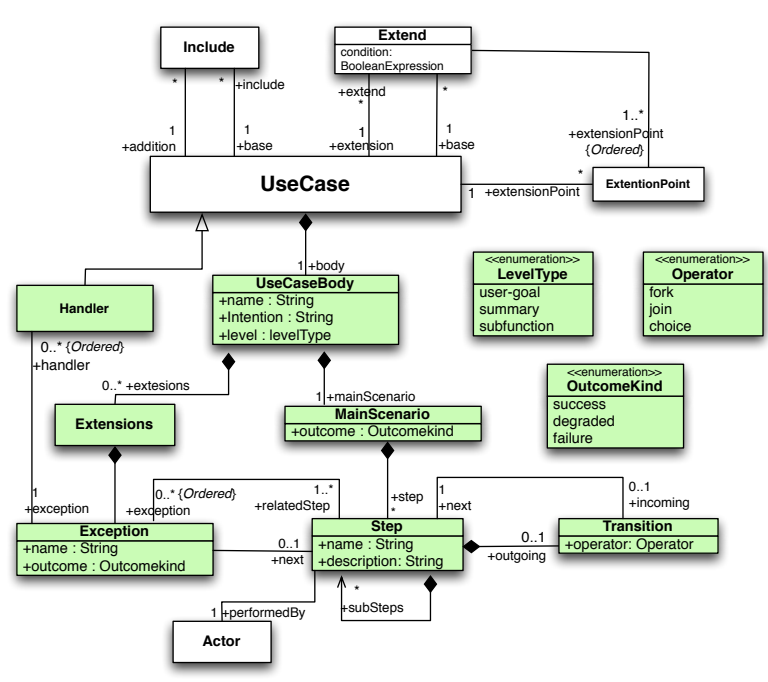

Figure 2: Metamodel of extended use case integrated in the metamodel of UML

After use case modeling, our algorithm consists in transforming each use case to a UML activity diagram $(T 1)$ and then extract the behavior of the each actor in a separate activity diagram $(T 2)$.

The approach used for (T1) is partially inspired form the work presented in (Gutiérrez et al., 2008). Authors in the cited article propose a method for representing functional requirements by automatically transforming use cases to activity diagrams. However, proposed use cases don't support the handling of identified exceptions. We propose a model transformation of extended use cases with handler to UML2 activity diagrams. The process of transforming extended use cases to activity diagrams consists in applying transformation rules enumerated bellow:

1. generate an activity for each use case,

2. generate an activity partition for each identified actor in the use case plus an additional one for the system,

3. generate an action for each Step in the main scenario,

4. add generated action to the activity partition of the actor identified in the performedBy attribute,

5. generate a decision node for each exception and additional activity for each handler,

6. generate an activity final node for each outcome in the use case labelled with a stereotype corresponding to the output kind,

7. link all generated elements using control flow.

To derive the behavior of each environment actor $(T 2)$, we propose an algorithm that derive these behavior while preserving the consistency of the global activity diagram. The algorithm extracts nodes and edges related to the same actor (based on activity Partitions) then links them together using control flow. To preserve the semantics of the global behavior described in the source activity diagram, events triggering the flow between actions and coming from the system or other actors are added to the targeted activity diagram. These events are represented with instances of the AcceptEventAction class (OMG, 2007). Thus, the flow between actions in the activity of a specific actor is conditioned with the reception of the corresponding event. Thus, the master rule to transform each activity group into a single activity diagram (AD) is defined as follows.

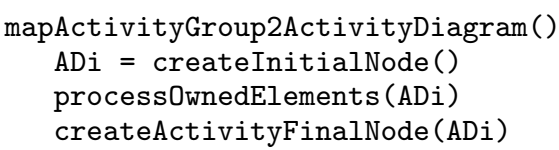

The result of applying our synthesis algorithm on the activity diagram generated from (T1) produce an activity diagram for each actor participating in the considered use case. To preserve 
interactions between actors, instances of the $A c$ ceptEventAction class are added, each time a transition linking two activities related to two different actors is detected.

\section{RELATED WORKS}

The work presented in (Almendros-Jimenez and Iribarne, 2004) describes an approach to translate use case-based functional requirements to activity charts. The source models are use cases diagrams with support of inclusion and generalization relationships. In (Gutiérrez et al., 2008), authors propose a model based approach to generate an activity diagram modeling the use case scenario. A functional requirement metamodel was proposed to represent the use case scenario with possible exceptions.

Mustafiz et. al (Mustafiz et al., 2009) propose an algorithm that transforms dependabilityfocused use cases with handlers into activity diagrams. The transformation takes textual use cases description as source to produce activity diagram model respecting the use case hierarchy source model. Our approach differs in the sense that we begin with informal requirements specification, namely extended use cases, apply a model-driven process to map requirements to activity diagrams, and then, automatically extract formal behavioral models of actors that interact with the system using our synthesis algorithm.

\section{CONCLUSION AND FUTURE WORK}

In this paper we introduce the UCM framework to ease the integration of formal verification techniques into software development. We have proposed a metamodel of extended use cases with handlers that address detected exceptions. Exceptional situations are less common and hence the behavior of the system in such situations is less obvious. Therefore, the proposed metamodel represents a good starting point for the identification of environment actors that might interact with the system. We have also proposed an approach to automatically synthesis environment entities behavior directly from constructed use cases. We have generated an activity diagram that describe the behavior of each use case using our model transformation rules. Then, we ex- tract the behavior of each actor participating to the activity in a separate activity diagram. The motivation behind this contribution is to ease the use of formal verification techniques by providing early context descriptions with enough precision to feed formal verification tools. To the best of our knowledge, there is no similar work dealing with this particular problem.

As a future work, we firstly plan to formalize requirement decomposition and formalization (the right side of figure 1). Secondly, we would like to evaluate the usefulness of introducing UCM into industrial practices as we did for CDL in (Dhaussy et al., 2009). And finally, we will integrate our approach in the complete verification process.

\section{REFERENCES}

Almendros-Jimenez, J. and Iribarne, L. (2004). Describing use cases with activity charts. Metainformatics, 3511 of LNCS. Springer:141-159.

Dhaussy, P., Auvray, J., De belloy, S., Boniol, F., and Landel, E. (2008). Using context descriptions and property definition patterns for software formal verification. In Workshop Modevva08,hosted by ICST 2008.

Dhaussy, P., Pillain, P.-Y., Creff, S., Raji, A., Traon, Y. L., and Baudry, B. (2009). Evaluating context descriptions and property definition patterns for software formal validation. MoDELS, LNCS 5795:438-452.

Dwyer, M. B., Avrunin, G. S., and Corbett, J. C. (1999). Patterns in property specifications for finite-state verification. ICSE, pages 411-420.

Gutiérrez, J., Nebut, C., Escalona, M., and Mejías, M. (2008). Visualization of use cases through automatically generated activity diagrams. MOD$E L S$.

Lamsweerde, A. V. (2009). Requirements engineering: From system goals to uml models to software specifications. Book.

Mustafiz, S., Kienzle, J., and Vangheluwe, H. (2009). Model transformation of dependability-focused requirements models. Proceedings of the 2009 ICSE Workshop on Modeling in Software Engineering.

Nebut, C., Fleurey, F., LeTraon, Y., and Jézéquel, J.-M. (2003). A requirement-based approach to test product families. 5th Intl. Workshop on Product Family Engineering (PFE-5).

OMG (2007). UML 2.1.2 superstructure. pages 1738. 